\title{
Für wen ist dieses Buch gedacht?
}

Wenn man sich den Titel des Buches ansieht, scheint die Antwort auf diese Frage klar zu sein: dieses Buch ist für Mitarbeiter in öffentlichen und wissenschaftlichen Bibliotheken gedacht, die sich im Rahmen ihrer Bibliotheksarbeit mit dem Thema Gaming befassen möchten. Dabei ist es egal, ob Sie Gaming-Experte sind oder nicht. Sie benötigen kein Vorwissen, um mit diesem Buch arbeiten zu können. Was Sie aber benötigen, ist die Offenheit für ein Thema, welches in seiner Breite aus Sicht der Bibliotheken immer noch zu selten diskutiert wird. Ob Sie nach der Lektüre des Buches im Bereich Gaming aktiv werden oder nicht, und wie diese potentiellen Aktivitäten dann aussehen, entscheiden Sie selber.

Auch wenn der Titel des Buches ausdrückt, dass es um Gaming in Bibliotheken geht, ist es für eine viel größere Zielgruppe gedacht. Ich wollte kein bibliothekarisches Basiswerk schaffen. Es geht nicht um die Mitarbeiter in Bibliotheken, sondern um das Thema Gaming in Bibliotheken. Die Mitarbeiter von Bibliotheken sind also nur ein Teil der Zielgruppe. Denn wenn wir uns mit dem Thema Gaming in Bibliotheken befassen, dann reden wir letztlich über verschiedene Akteure, die das Thema in der jeweiligen Bibliothek gemeinsam umsetzen werden. Es wird Bibliotheken nur sehr selten gelingen, alleine ein nachhaltig erfolgreiches Gaming-Konzept zu entwickeln und zu realisieren. Vielmehr werden interdisziplinäre Netzwerke benötigt. Die Bibliothek mag bestimmte Ressourcen und Knowhow zu Verfügung stellen können. Es wird aber ebenso wichtig sein, ein umfassendes Gaming-Netzwerk aufzubauen. $\mathrm{Zu}$ diesem Netzwerk gehören dann natürlich die Mitarbeiter der Bibliothek aber ebenso Gamer, Eltern, Lehrer, Mitarbeiter der Stadt, potentielle Sponsoren etc. Es gibt also eine Vielzahl an Personen, die potentiell Kontakt mit dem Thema haben werden. Damit diese Netzwerke existieren können, ist es wiederum wichtig, dass alle Beteiligten ein Gespür für das Thema Gaming und seine Nutzungsmöglichkeiten in Bibliotheken bekommen. Deshalb ist dieses Buch für alle Menschen gedacht, die sich mit der Frage beschäftigen möchten, wie Bibliotheken sich dem Thema Gaming nähern könnten.

Dieses Buch ist also für eine breite Zielgruppe gedacht. Deshalb meine Bitte an alle Bibliotheksmitarbeiter: geben Sie dieses Buch weiter! Diskutieren Sie die Inhalte mit Eltern, Lehrern, Gamern etc. Versuchen Sie, sich gemeinsam zu überlegen, was die für Ihre Bibliothek passenden nächsten Schritte sein könnten. Und wenn Sie dann die eine oder andere Aktivität umgesetzt haben, geben Sie mir bitte ein Feedback. Teilen Sie Ihre Ideen und Erfahrungen mit anderen Bibliotheken bzw. anderen Institutionen. Dieser Austausch ist sehr wichtig, denn nur so kann das Thema in den Bibliotheken an Bedeutung gewinnen. Keine Bibliothek ist wie die andere. Früher oder später werden Sie mit Problemfeldern konfrontiert werden, bei denen Sie nicht wissen, wie Sie diese lösen können. Die Erfahrung zeigt aber, dass es in den allermeisten Fällen irgendwo jemanden gibt, der das gleiche Problem hatte und eine dazu passende Lösung gefunden hat. Leider werden in unseren Breitengraden gerne Erfolge und nur selten Probleme bzw. Niederlagen kommuniziert. Aber genau das müssen Sie unbedingt tun. Und nebenbei lernen Sie auf diesem Weg bereits Ihre erste wichtige GamerLektion: Try and Fail. Haben Sie also keine Angst - Gamer sind es gewohnt, Fehler zu machen und aus den Fehlern zu lernen. Und Sie sind es ebenso gewohnt, Ihr Wissen aktiv zu teilen, damit alle mitspielen können. Je früher Sie also damit anfangen, desto erfolgreicher werden Sie sein. Und je mehr Menschen sich in Ihrem Umfeld mit dem Thema befassen, desto besser für Ihre Bibliothek. 


\section{Was dieses Buch leisten kann}

In diesem Buch geht es um Gaming und was Bibliotheken damit machen können. Wenn Sie dieses Buch lesen und damit arbeiten, können Sie eine individuelle Gaming-Strategie für Ihre Bibliothek entwickeln. Sie werden zudem erfahren, was mit Gaming eigentlich gemeint ist, welchen Einfluss Gaming auf das Lernen und Arbeiten in der Zukunft hat, wie diese „Gamer“ eigentlich aussehen, was man beachten sollte, wenn man in diesem Bereich aktiv werden sollte und natürlich welche Hard- und Software es gibt. Dieses Buch ist also als Einstieg gedacht. Es ist aber so konzipiert, dass Sie zumindest Teile davon auch noch verwenden können, wenn Sie schon länger aktiv und erfolgreich sind. Dieses Buch zeigt auch eventuelle Problemfelder auf und versucht, Lösungsansätze zu formulieren. Selbst dann, wenn Sie sich mit dem Thema aus welchen Gründen auch immer nicht aktiv in Ihrer Bibliothek beschäftigen können oder dürfen, kann dieses Buch helfen, die Thematik besser zu verstehen. Und es geschehen ja noch Zeichen und Wunder, und wenn wir irgendwann erleben, dass sich der Bundespräsident bzw. die Bundespräsidentin als GamerIn outen, werden wir Großartiges erleben :-)

Dieses Buch ist ein Praxisbuch, d.h. es wird nicht um eine philosophisch-soziologische Diskussion zum Thema Gaming gehen. Was kann bzw. sollte man mit diesem Buch machen? Lesen! Aber bitte nicht nur einmal. Dies ist kein klassisches Werk der Romantik, kein Essay oder eine Polemik. Es ist ein Arbeitsbuch. Also bitte arbeiten Sie damit. Sie dürfen, ja Sie sollen in das Buch eigene Kommentare einfügen. Das ist es, was ich mit meinen Büchern immer wieder mache. Jemand, der meine Bücher, aber mich nicht persönlich kennt, wird meine Bücher für individuelle Gesamtkunstwerke halten. Ich male in ihnen herum, markiere Textstellen, klebe Seiten rein etc. Also bitte: nutzen Sie dieses Buch als ein Werkzeug. Dieses Buch sollte nicht in einem Regal verschwinden. Dort verliert es seine Kraft - aus Sicht eines Gamers hat jedes Werkzeug eine eigene Kraft, die es verliert, wenn es nicht genutzt wird :-)

Dieses Buch ist aber nicht die Lösung für alle Probleme, die sich aus der Nutzung bzw. Beschäftigung mit Gaming ergeben können. Wie bereits zuvor erwähnt, gibt es mit Sicherheit Problemstellungen, die ich übersehen habe, oder die niemand einplanen konnte. Hier ist Ihr Feedback wichtig. Wenn Sie ein Problem haben, welches in diesem Buch nicht besprochen wird, oder wenn Sie vielleicht sogar eine Lösung für ein Problem gefunden haben, dann bitte senden Sie mir eine Mail an christoph. deeg@googlemail.com oder teilen Sie Ihr Wissen und Ihre Fragen auf einer der eben genannten Plattformen.

Dieses Buch soll Ihnen den Einstieg in das Thema ermöglichen. Sie werden eine Vielzahl an Informationen vorfinden. Letztlich müssen Sie selber entscheiden, für welchen Weg Sie sich entscheiden. Natürlich bekommen Sie auch Hintergrundwissen zum Thema Gaming. Selbst wenn Sie das Thema nur in einem kleinen Rahmen behandeln wollen, ist es sinnvoll, einen ersten Überblick über den gesamten Themenkomplex zu bekommen. Aber letztlich ist es ein Praxisbuch. Deshalb bekommen Sie neben Informationen und Tipps auch kleine Aufgaben, die Ihnen helfen sollen, das Thema in der Realität kennen zu lernen. Umsetzen müssen Sie diese Aufgaben natürlich selber :-) Und haben Sie keine Angst, Fehler zu machen - das gehört wie gesagt dazu.

Dieses Buch basiert auf der Idee, dass sich eine Bibliothek intensiv mit dem Thema Gaming in allen seinen Facetten beschäftigen möchte. Mit Sicherheit möchten und können sich nur die wenigsten Bibliotheken mit diesem Thema in aller Breite beschäftigen. Das heißt letztlich müssen Sie selbst entscheiden, welche der in diesem Buch niedergeschriebenen Ansätze Sie verfolgen möchten. 


\section{Was dieses Buch nicht leisten kann}

Dieses Buch ist natürlich perfekt. Es macht Sie zum absoluten Gaming-Superhelden :-) Ok, das stimmt nicht ganz. Dieses Buch kann nur einen ersten Einstieg in das Thema ermöglichen. Ein allumfassendes Werk scheitert an der Komplexität des Themas und der Tatsache, dass sich die Welt des Gamings kontinuierlich und sehr schnell weiterentwickelt. Ein Beispiel: In diesem Buch werde ich u.a. die beiden Konsolen XBOX360 und Playstation3 näher beschreiben. Zum Zeitpunkt der Veröffentlichung dieses Buches wird aber wahrscheinlich schon die nächste Generation an Konsolen auf den Markt gekommen sein. Dieses Buch ist deshalb kein Trendbuch. Aktualität ist aber ein wichtiges Erfolgskriterium. Aus diesem Grund gibt es die begleitenden Plattformen, wie z. B. meinen Blog (www.christoph-deeg.de) oder die Seite des Netzwerkes „games4culture“ (www.games4culture.de).

Aber selbst wenn Sie sich kontinuierlich mit dem Buch befassen, wird es einige wichtige Aufgaben nicht lösen können. So wird es auf keinen Fall das eigene Ausprobieren ersetzen können. Auch wenn dieses Buch ein Praxisbuch ist, ist es „nur“ Theorie. Und auch wenn Sie es 100x durchgelesen haben, werden Sie mit an Sicherheit grenzender Wahrscheinlichkeit die Welt des Gamings nicht verstanden haben. Egal wie Sie es drehen und wenden, Sie müssen selber zum Gamer werden. Sie müssen spielen. Ohne das geht es nicht. Mir ist bewusst, dass ich u.U. einiges von Ihnen verlange, aber wie wollen Sie ein erfolgreiches Angebot entwickeln und umsetzen, wenn Sie nicht wissen, was Spielen bedeutet? Nun mögen Sie einwenden, dass Sie in Ihrer Bibliothek eine Vielzahl an Medien bzw. Inhalten anbieten, die Sie selber nicht nutzen. Aber Gaming ist etwas komplett anderes. Im Lauf der nächsten fast 200 Seiten werden Sie - hoffentlich - verstehen, dass Gaming mehr ist als nur ein weiteres Medium bzw. ein weiterer Inhalt. Gaming ist zugleich eine neue Kultur, ein neues Denkmodell, eine neue Form der Kultur- und Wissensvermittlung, eine neue Form der Integration digitaler Medien in die Bibliotheksarbeit und eine starke Vernetzung mit den Themen Social Media und Mobile Internet. Das alles bedeutet, dass Sie zumindest am Anfang Zeit für das eigene Ausprobieren investieren müssen. Aber keine Angst - in der Regel macht es sehr großen Spaß :-)

Dieses Buch ist von einem Menschen geschrieben worden, der selber Gamer ist und zudem in diesem Bereich arbeitet. Es ist also kein neutrales Buch. Es ist ein Buch für die Menschen, die Gaming wollen. Es gibt deshalb keine Auseinandersetzung über die Vor- und Nachteile der Nutzung von Games. Und fragen Sie sich selbst: Wann haben Sie das letzte Mal über die Vor- und Nachteile des Lesens von Büchern diskutiert? Games sind nicht besser aber auch nicht schlechter als Bücher. Wenn Sie nach Gründen suchen, warum Gaming vielleicht doch gefährlich und sinnlos ist, dann sollten Sie jetzt mit dem Lesen aufhören.

Und wenn Sie nach der Lektüre des Buches und einer Phase des Ausprobierens der Meinung sind, dass Gaming an sich sinnlos ist und nur dafür benutzt werden sollte, um damit junge Zielgruppen in die Bibliothek zu locken, damit sie dann Bücher lesen, dann sollten Sie in diesem Bereich nicht mit eigenen Aktivitäten starten. Denken Sie immer daran: Die Welt des Gamings (und dies gilt letztlich für alle digitalen Medienformen wie Social Media, Mobile Internet etc.) hat sich wunderbar ohne Bibliotheken entwickelt. Bibliotheken können unglaublich viel mit dem Thema Gaming machen. Und sie können natürlich neue Zielgruppen erreichen. Ihnen muss aber auch klar sein, dass Sie dafür die Kultur Ihrer Zielgruppe akzeptieren müssen. 